\title{
The Impact of Parental and Peer Social Support on Dating Violence Perpetration and Victimization Among Female Adolescents: A Longitudinal Study
}

\author{
Tara N. Richards, PhD \\ University of Baltimore \\ Kathryn A. Branch, PhD \\ University of Tampa \\ Katherine Ray, BS \\ University of Tennessee at Chattanooga
}

\begin{abstract}
Little is known about the role social support may play in reducing the risk of adolescent dating violence perpetration and victimization. This study is a longitudinal analysis of the independent impact of social support from friends and parents on the risk of emotional and physical dating violence perpetration and victimization among a large sample of female youth $(n=346)$. Findings indicate that $22 \%$ of the sample indicated perpetrating physical dating violence against a partner, whereas almost $16 \%$ revealed being the victim of physical dating violence; $34 \%$ of the sample indicated perpetrating emotional dating violence against a partner, whereas almost $39 \%$ revealed being the victim of emotional dating violence. Negative binomial regression models indicated that increased levels of support from friends at Time 1 was associated with significantly less physical and emotional dating violence perpetration and emotional (but not physical) dating violence victimization at Time 2. Parental support was not significantly related to dating violence in any model. Implications for dating violence curriculum and future research are addressed.
\end{abstract}

Keywords: dating violence victimization; dating violence perpetration; social support; adolescents

$\mathrm{R}$ esearch has documented that dating violence (DV) is a significant issue in the lives of many adolescents (Lewis \& Fremouw, 2001; O'Keefe, 1998). Much of this research has focused on physical DV and has demonstrated a link between physical DV and negative emotional and physical outcomes (Carlson, 1987; Muñoz-Rivas, Graña, O’Leary, \& González, 2007; Silverman, Raj, Mucci, \& Hathaway, 2001). Prior research has also demonstrated that contrary to adult intimate partner 
violence (IPV), adolescent females are disproportionately involved in DV as both victims and perpetrators (Foshee et al., 1996; Richards \& Branch, 2012; Swahn, Simon, Arias, \& Bossarte, 2008). Comparatively, less research has focused on nonphysical DV such as emotional and/or psychological DV, although the limited existing studies suggest that nonphysical DV may cause as much or more long-term damage than physical DV (O'Leary, 1999).

At the same time, although much attention has focused on strategies to combat DV (Lewis \& Fremouw, 2001) as well as the risk factors associated with both perpetration and victimization (Raiford, Wingood, \& Diclemente, 2007; Silverman et al., 2001), there has been a paucity of investigations regarding characteristics that may prevent $D V$ perpetration and victimization. One such factor may be social support. Social support has been extensively documented as a positive measure against IPV among adult women (Branch, 2008; Carlson, McNutt, Choi, \& Rose, 2002; Dobash, Dobash, Cavanaugh, \& Lewis, 1998; Larance \& Porter, 2004; Thompson et al., 2000) and limited cross-sectional research has demonstrated an association between higher levels of social support from friends and lower levels of physical DV perpetration and victimization among adolescent girls (but not boys; Richards \& Branch, 2012). This study aims to extend our understanding of the impact of social support on DV among adolescent females by investigating whether there is a longitudinal association between parental and/or peer social support and later DV perpetration and/or victimization among adolescent girls. This research will also expand on prior works by including separate measures of physical and emotional DV, allowing for the exploration of any differential effects of social support on each type of violence.

\section{LITERATURE REVIEW}

Research has documented that violence in teen dating relationships exists in multiple forms (Lavoie, Robitaille, \& Hebert, 2000; Sears, Byers, Whelan, \& Saint-Pierre, 2006). Traditionally, research on teen dating relationships has focused on physical abuse. More recent efforts have begun to focus specifically on adolescent experiences of emotional abuse (Abowitz, Knox, \& Zusman, 2010; Lavoie et al., 2000). Such research suggests that emotional aggression may have a more severe impact on victims than physical aggression and may be more widespread than physical abuse (Linder, Crick, \& Collins, 2002). Sears et al. (2006) suggests that more girls and boys are using emotional abuse as a tool to gain power and control in a dating relationship than in the past because physical abuse is no longer viewed by teens as an acceptable behavior.

Extant literature indicates that both boys and girls use physically and emotionally abusive behaviors in their dating relationships (Abowitz et al., 2010; Lavoie et al., 2000). Teens may lack the skills and experience needed to successfully respond to feelings and emotions, resolve arguments, and reach compromise in conflicts. As a result, teens may fall back on prescribed gender role expectations. Traditionally, boys are not encouraged to discuss feelings or problems (Maccoby, 1998). Therefore, boys may have limited options in how to handle emotions such as anger and jealousy. In contrast, female gender roles prescribe that girls express their emotions verbally rather than through physical force (Maccoby, 1998). Thus, accurate depictions regarding the complex nature of violence in dating relationships must include examinations of both physical and emotional violence, especially with adolescent females (Lavoie et al., 2000). 


\section{Physical Dating Violence}

Almost three decades ago, Makepeace's (1981) seminal study uncovered adolescent dating violence as a pervasive problem. Recent nationally based samples have revealed that $8 \%-12 \%$ of adolescents have been physically victimized by a dating partner (Ackard, Neumark-Sztainer, \& Hannan, 2003; Carver, Joyner, \& Udry, 2003; Howard \& Wang, 2003; Roberts \& Klein, 2003). Some research on adolescents' use of violence has suggested that girls are more physically abusive toward their dating partners than boys (Foshee et al., 1996; O'Keefe, 1997). Many times, however, girls' use of physical aggression is perceived as "joking around" because females rarely cause physical harm to adolescent males (Sears et al., 2006, p. 1198). Other research suggests that physical DV victimization disproportionately affects adolescent girls. For example, Silverman et al. (2001) found that female adolescents in their sample experienced a 3-6 times greater risk of physical DV victimization compared to their male counterparts. Prior literature also suggests that, although most intimate violence between male and female dating partners can be categorized as mutual, after controlling for self-defense, females suffer greater rates of physical victimization (Foshee et al., 1996). Furthermore, extant studies have suggested that boys are more likely to use severe physical violence than girls (e.g., Bennett \& Fineran, 1998) and that $10 \%$ of all intentional injuries to adolescent girls are the results of a male dating partner (Silverman et al., 2001).

\section{Emotional Dating Violence}

Emotional abuse is behavior that is designed to control, psychologically subjugate, or harm the recipient (Abowitz et al., 2010). Examples of emotional abuse include manipulation, verbal abuse, constant criticism, intimidation, name calling, and a refusal to be pleased. Emotional abuse may exist alone but it is often inflicted in conjunction with other forms of abuse (Abowitz et al., 2010). Recent studies of dating violence demonstrate that psychological/emotional DV may occur at higher rates than physical or sexual violence. For example, Sears and Byers (2010) found that although 29\% of the female youth in their sample experienced physical violence, $62 \%$ reported experiencing emotional violence. Victims of emotional abuse often report that the abuse has a significant impact on multiple facets of well-being including mental health, relationships, and the ability to adjust to change (Chen, Williams, Fitness, \& Newton, 2008; Gavin, 2011). In addition, research suggests that emotional abuse is as damaging to victims' health as physical abuse (O’Leary, 1999) or may be even more destructive (Gavin, 2011).

Emotional abuse does not have the same visibility as sexual or physical abuse but it can erode an individual's self-confidence, sense of self-worth, and self-concept (Rees, 2010). Childhood and early adolescence is the period that an individual should begin to feel empowered and have influence over his/her life events (Zimmerman, 1995). Exposure to emotional/psychological abuse during this period may alter the development of personal control and potentially make one more susceptible to negative experiences in later adolescence and adulthood (Berenson \& Anderson, 2006).

\section{Impact of Social Support}

Social support is generally defined as information that makes an individual believe that he/ she is "cared for, loved, esteemed, and valued and is a member of a network of common and mutual obligation" (El-Bassel, Gilbert, Rajah, Foleno, \& Frye, 2001, p. 247). Prior 
literature suggests that social support works differently for male and female adolescents (Colarossi \& Eccles, 2003; Malecki \& Demaray, 2003). Compared to male youth, female youth are significantly more likely to pursue social support (Colarossi \& Eccles, 2003; Malecki \& Demaray, 2003) and perceive higher levels of social support (Malecki \& Demaray, 2003). Prescribed gender roles and gendered peer culture may explain, at least in part, these differences. Maccoby (1998) suggests that girls' peer culture encourages girls to have dyadic relationships that are characterized by empathetic understanding, intimacy, self-disclosure, and exclusivity. Comparatively, boys' peer culture encourages boys to have large circles of less intimate friendships (Maccoby, 1998; Underwood \& Rosen, 2009). As a result of these two different peer cultures, adolescent boys and girls are socialized to have different expectations about commitment, loyalty, and understanding from friends (Clark \& Ayers, 1993; Kuttler, La Greca, \& Prinstein, 1999).

Research suggests that adolescent females report higher levels of support from friends than from parents (Malecki \& Demaray, 2003), whereas adolescent males report higher levels of support from parents than from peers (Frey \& Rothlisberger, 1996). In addition, during adolescence, youth attempt to develop a personal identity separate from their parents so the significant role of parents in the lives of youth shifts to youths' peer group (Giordano, Longmore, \& Manning, 2006; Haynie, Steffensmeier, \& Bell, 2007; Krosnick \& Judd, 1982). Although research suggests that parents retain influence over their teens during adolescence (Eiser, Morgan, Gammage, \& Gray, 2011; Martino, Ellickson, \& McCaffrey, 2009), youth place a high value on their peer friendships and, as a result, adolescents are strongly influenced by friends (Giordano et al., 2006; Warr, 2002).

Recent research has suggested that there are qualitative differences in the protective role of social support from friends and social support from family. Richards and Branch (2012) analyzed the protective impact of social support from friends and parents against physical DV perpetration and victimization among adolescent males and females. Findings indicated that friends' social support was significantly associated with a decrease in physical DV perpetration and victimization for females (but not males). Comparatively, parental social support was not significantly associated with physical DV. Although Richards and Branch's prior research uncovered important information, it also had several limitations. First, the research used cross-sectional data, and thus, could not establish a causal relationship between social support and DV because the temporal relationship between these variables could not be determined. Also, the study was solely focused on physical DV, not emotional DV, although prior research demonstrates that emotional DV is a more widespread problem than physical DV.

\section{THE PRESENT STUDY}

This study builds on the existing literature indicating that social support serves as a protective factor against IPV for adult women (Branch, 2008) and the cross-sectional research indicating that social support from friends is associated with a decreased likelihood of physical DV perpetration and victimization for adolescent females (Richards \& Branch, 2012). This investigation is the first research to date that has examined the longitudinal impact of social support on DV victimization and perpetration for female youth. Specifically, this research investigates whether higher levels of parental and/or peer social support at Time 1 serves as a buffer against physical and emotional DV victimization and/ or perpetration at Time 2 net of other important control variables. 


\section{METHODS}

\section{Data and Sample}

The data for this study were drawn from Wave I (2001) and Wave II (2002) of the Toledo Adolescent Relationship Study (TARS), a 5-year investigation regarding the context and significance of adolescent relationships (e.g., family, peers, and dating partners; Giordano, Longmore, \& Manning, 2001). The TARS data consists of a stratified, random sample ( $n=$ 1,316) drawn from all 7th, 9th, and 11th grade youth residing in Lucas County in the fall of 2000. Participants were identified using public and private school enrollment records for Lucas County, Ohio; school attendance was not a requirement for inclusion in the research. Structured, in-home interviews were conducted using laptop computers preloaded with the questionnaire. Interviewers administered the demographic questions while participants operated the computer independently to complete personal survey questions. This research included data from all female participants who indicated at Wave II that they had previously dated (within the last year) or were currently in a dating relationship $(n=346)$.

\section{Independent Variables (Measured at Wave I)}

Social support from parents and friends were independently assessed for each participant using two scales from Richards and Branch (2012). The level of parental social support was assessed by a five-item scale asking participants how much they agreed or disagreed with the following statements about their parents: My parents often ask me what I am doing in school; My parents give me the right amount of affection; I can go to my parents with concerns about the opposite sex; I feel close to my parents; and My parents trust me. The level of friends' social support was assessed by a five-item scale asking participants how much they agreed or disagreed with the following statements about their friends: I can tell them private things and I know they won't tell other people; They care about me; My friends make me feel good about myself; I feel comfortable talking with my friends when I have a problem; and I feel close to my friends. For both scales, the response format was $0=$ strongly disagree, $1=$ disagree, $2=$ neither agree nor disagree, $3=$ agree, $4=$ strongly agree. Answers were summed for a total score. Internal consistency reliability analysis for each of the measures indicated that the two social support scales had acceptable Cronbach's alphas: parental social support $(\alpha=.79)$ and friend social support $(\alpha=.76)$.

Family violence was assessed using items from the Revised Conflict Tactics Scale (Straus \& Gelles, 1990). Participants were asked how often during a disagreement they do the following things to their parents and their parents do the following things to them: call them names or insult them; push, slap, or hit them; yell at them? Each item was coded from $0=$ never to $5=$ two or more times a week. Adolescents' responses were summed yielding a measure of perpetration of family violence $(\alpha=.60)$ and victimization of family violence $(\alpha=.68)$.

Several demographic variables as well as behavioral risk factors were included in the analyses as control variables. Demographic variables included age in years, race/ethnicity, and average grade earned (measured on a 9-point scale from mostly $F$ 's $=1$, mostly $D$ 's and F's $=2$, to mostly A's $=9$ ). Behavioral risk factors included participant alcohol and drug use and participation in delinquency. Alcohol and drug use was assessed by two dichotomous variables coded 0 if participants reported never using alcohol or drugs and 1 if the respondent reported ever using alcohol or drugs. Delinquency was measured by 
asking participants how often, in the past 12 months, they had participated in the following: damaged or destroyed property; stolen something worth more than $\$ 50$ dollars; attacked someone with the idea of seriously hurting them; sold drugs; and broken into a building or vehicle. Each item was coded so that $0=$ never to $8=$ more than once a day. Adolescents' responses were summed yielding a measure of involvement in delinquency $(\alpha=.70)$.

\section{Dependent Variables (Measured at Wave II)}

Each respondent's experiences with physical and emotional DV perpetration and victimization were obtained using items from the Revised Conflict Tactics Scale (Straus \& Gelles, 1990). Physical DV perpetration was assessed by a four-item scale asking participants how often they had done the following things to their current (or most recent) boyfriend/girlfriend: thrown something at him/her; pushed, shoved, or grabbed him/her; slapped him/her in the face or head with an open hand; hit him/her? Physical DV victimization was assessed by a four-item scale asking participants how often their current (or most recent) dating partner had done the following things: thrown something at you; pushed, shoved, or grabbed you; slapped you in the face or head with an open hand; hit you? The response format was $0=$ never, $1=$ hardly ever, $2=$ sometimes, $3=$ often, $4=$ very often. Answers were summed for a total score. Internal consistency reliability analysis indicated the DV scales had strong Cronbach's alphas: physical DV perpetration $(\alpha=.94)$ and physical DV victimization $(\alpha=.80)$.

Emotional DV perpetration was assessed by four-item scale asking participants how often they had done the following things to their current (or most recent) boyfriend/girlfriend: ridiculed or criticized their values or beliefs; put down their physical appearance; put them down in front of other people? Emotional DV victimization was assessed by a three-item scale with questions asking participants how often their current (or most recent) dating partner had done the following things: Ridiculed or criticized your values or beliefs; put down your physical appearance; put you down in front of other people? The response format was $0=$ never, $1=$ hardly ever, $2=$ sometimes, $3=$ often, $4=$ very often. Answers were summed for a total score. Internal consistency reliability analysis indicated the DV scales had acceptable Cronbach's alphas: emotional DV perpetration $(\alpha=.75)$ and emotional violence victimization $(\alpha=.78)$.

\section{ANALYTIC STRATEGY}

To explore whether social support from parents and/or peers mitigates emotional and/or physical DV perpetration and/or victimization among adolescent girls, a series of negative binomial regression models were conducted. Negative binomial regression is well suited for dependent variables with an excess of zeros (such as DV perpetration or victimization) and a substantial positive skew (a minority of respondents reporting very high levels of DV perpetration or victimization). The results of negative binomial regression models are easily understood by exponentiating coefficients so that a standard deviation increase in a youth's perception of social support from friends or family is associated with a percentage increase or decrease in either DV perpetration or victimization. A series of negative binomial regression models were estimated to explore the impact of social support at Time 1

(net of other covariates) on the likelihood of physical and emotional DV perpetration and victimization at Time 2 . 


\section{RESULTS}

Descriptive statistics revealed that the average age for respondents was approximately 15.66 years old $(S D=1.66)$. Almost $46 \%$ of the sample was in 11th or 12 th grade, $31 \%$ were in 9th or 10th grade, and $23 \%$ were in 7 th or 8 th grade. Regarding race/ethnicity, $67 \%$ of the sample reported being White and 33\% reported being non-White. Non-White participants included the following: 63\% Black; 20\% Hispanic; 2\% American Indian and Asian, respectively; and $13 \%$ other race/ethnicities. When asked about their average grades, approximately $15 \%$ of participants indicated earning mostly A's, $29 \%$ A's and B's, $8 \%$ mostly B's, $24 \%$ mostly B's and C's, $10 \%$ mostly C's, $10 \%$ mostly C's and D's, $2 \%$ mostly D's, $3 \%$ mostly D's and F's, and $1 \%$ earning mostly F's. Almost $22 \%$ of the sample indicated perpetrating physical DV against a partner while almost $16 \%$ revealed being the victim of physical DV. Comparatively, almost $34 \%$ of the sample indicated perpetrating emotional DV against a partner while almost $39 \%$ revealed being the victim of emotional DV. Similar to past research, most adolescents who reported experiencing DV were involved in mutual violence. Specifically, of the 103 girls who reported perpetrating emotional DV, 77\% also reported experiencing emotional DV victimization. Comparatively, of the 46 female youth who reported perpetrating physical DV, $61 \%$ also reported experiencing physical DV victimization.

Turning to the multivariate analyses, the results of two negative binomial regression models, one predicting physical DV perpetration and one predicting emotional DV perpetration using the measures of social support and the control variables, are presented in Table 1. Results indicate friends' social support is significantly related to lower levels of physical DV perpetration for adolescent females. For female youth, each unit increase in the friend's social support measure $(0=$ strongly disagree to $4=$ strongly agree $)$ is associated with an $18 \%$ decrease in the perpetration of physical DV. Parental social support, however, is not significantly associated with DV perpetration for female youth. In addition, female youth who reported higher average grades are less likely to perpetrate physical DV compared to those who reported lower average grades. On the other hand, female youth who experience more family violence from parents are significantly more likely to perpetrate physical DV compared to female adolescents who experience less parental violence. Specifically, higher levels of parental violence are associated with an $11 \%$ increase in physical DV perpetration. Also, non-White female youth are approximately twice as likely to perpetrate physical DV compared to White female youth. Regarding emotional DV perpetration, only friends' social support is significantly associated with perpetration for adolescent females. Similar to physical DV victimization, each unit increase in the friend's social support measure $(0=$ strongly disagree to $4=$ strongly agree $)$ is associated with an $18 \%$ decrease in the perpetration of emotional DV among female youth.

Turning to the second set of multivariate analyses, two negative binomial regression models, one predicting physical DV victimization and one predicting emotional DV victimization using the measures of social support and the control variables, are presented in Table 2. Results indicate that neither friends' nor parents' social support is significantly related to physical DV victimization for adolescent females. Similar to the results for physical DV perpetration, female youth who reported higher average grades were less likely to experience physical DV victimization compared to those who reported lower average grades. Deviating from the physical DV perpetration model, female youth who experienced more family violence from parents were no more at risk for physical DV victimization than female adolescents who experienced less parental violence. Non-White female youth were $37 \%$ 


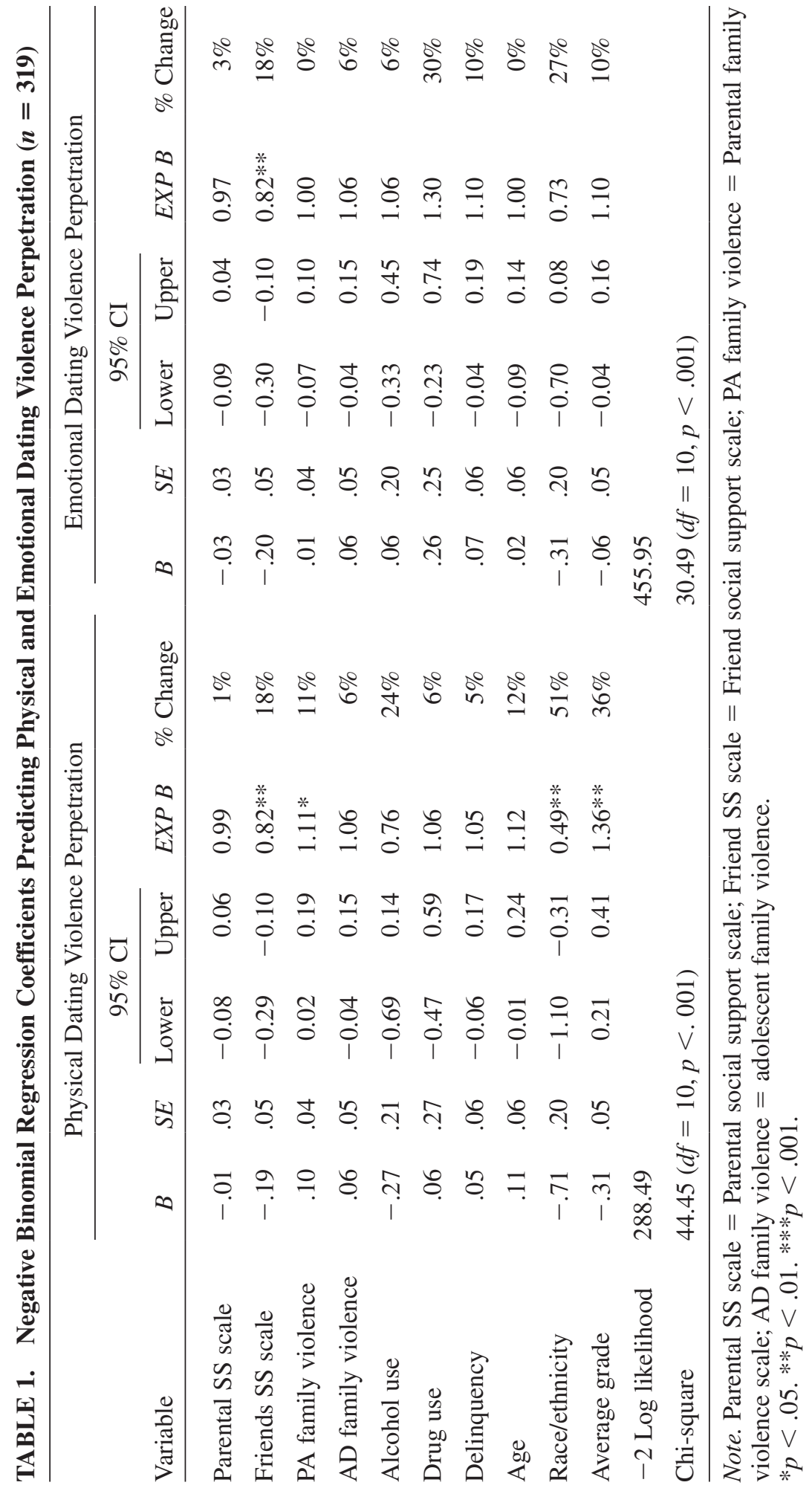




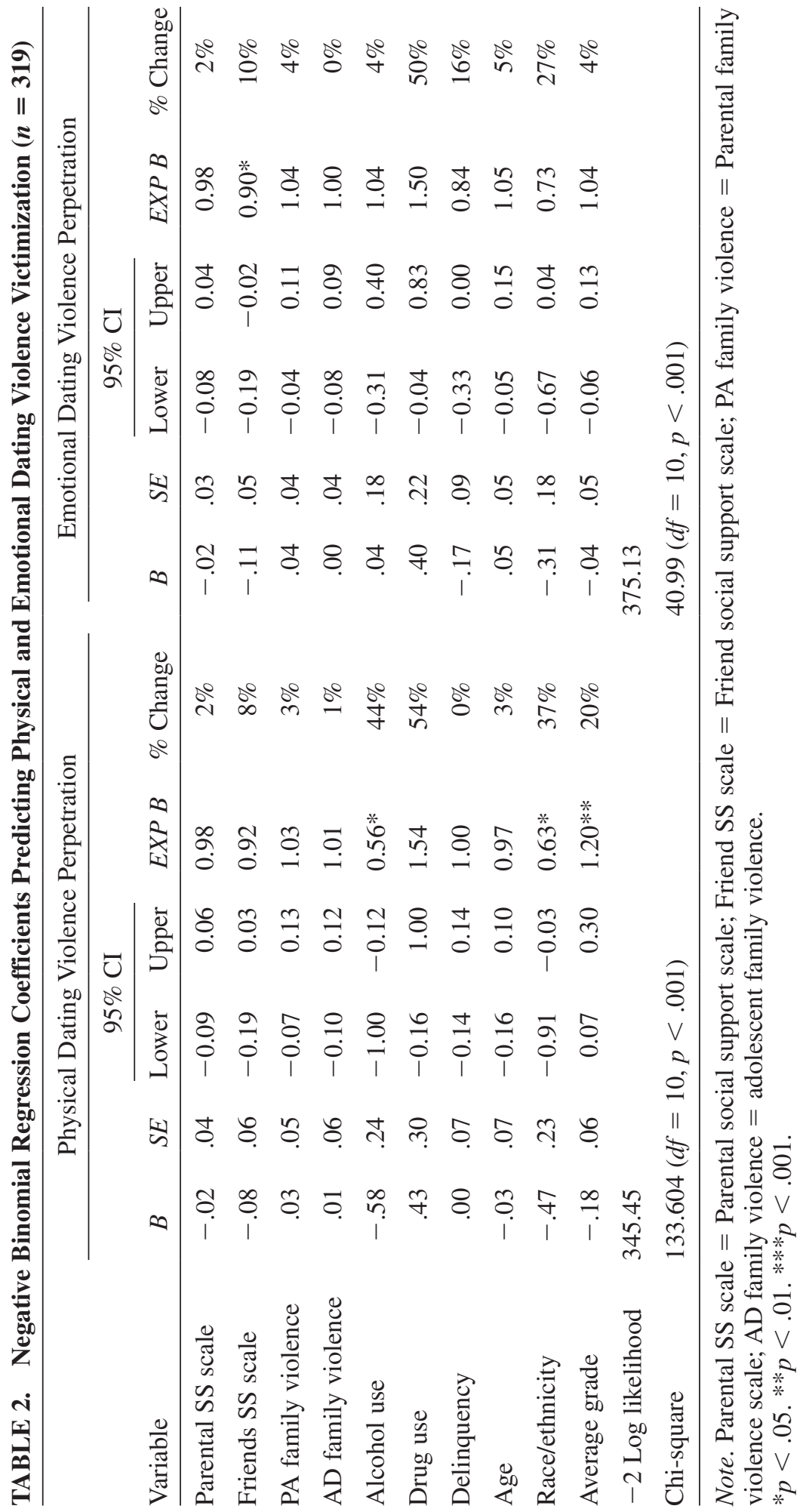


more as likely to experience physical DV victimization compared to White female youth. Regarding emotional DV perpetration, only friends' social support is significantly associated with victimization for adolescent females. Each unit increase in the friend's social support measure $(0=$ strongly disagree to $4=$ strongly agree $)$ is associated with a $10 \%$ decrease in the likelihood of experiencing emotional DV victimization among female youth.

\section{DISCUSSION}

The goals of this research were to longitudinally investigate (a) the impact of parental and/or peer social support on later DV perpetration and victimization among female adolescents and (b) to explore any differential effects of social support for emotional violence and physical violence. Across physical and emotional violence, more participants reported perpetrating physical violence and being victims of emotional violence. Consistent with prior literature (O'Leary \& Slep, 2012), findings from this study indicate that girls' perpetration rates for physical violence (22\%) are higher than their rates of victimization (16\%). Counter to extant research (Sears et al., 2006), girls' rates of emotional violence perpetration (34\%) were lower than their rates of victimization (39\%). In addition, consistent with prior literature (O’Leary \& Slep, 2012; Vézina \& Hébert, 2007), most girls who reported experiencing victimization or engaging in violence against a partner reported mutual violence. Specifically, of the 103 girls who reported perpetrating emotional DV, 77\% also reported experiencing emotional DV victimization. Comparatively, of the 46 female youth who reported perpetrating physical DV, $61 \%$ also reported experiencing physical DV victimization.

Negative binomial regression models revealed that for adolescent females, social support from friends at Wave I was significantly related to lower levels of both physical DV perpetration and emotional DV perpetration at Wave II. Comparatively, social support from parents was not significantly associated with either physical or emotional DV perpetration by female youth. These findings are consistent with cross-sectional research from Richards and Branch (2012) demonstrating that social support from friends but not parents is associated with a reduction in the perpetration of physical DV among female youth. The present results also exhibit evidence that this relationship holds true for emotional violence perpetration. Such findings are consistent with prior literature demonstrating that female youths' help-seeking behavior regularly includes the use of friends as social support providers, and less often employs assistance from families (Schonert-Reichl \& Muller, 1996).

Turning to the victimization models, results indicate that neither friends' nor parents' social support was significantly related to physical DV victimization for adolescent females; however, friends' social support was significantly associated with lower levels of emotional DV victimization. Such findings are partially consistent with cross-sectional research from Richards and Branch (2012), which demonstrates that parental social support is not influential on physical DV victimization but deviates from their findings that friends' support is associated with lower physical DV victimization. The present results indicate a need for systematic research on how friends' social support impacts adolescent DV victimization longitudinally. It may be that girls with higher levels of friends' social support escape physical violence in the short term but over time may be isolated from their friends by an abusive partner dispelling the positive effects of friends' social support. In addition, Richards and Branch suggest that social support from friends may only be impactful on physical DV victimization if friends are able to identify signs of DV and obtain resources for victims/potential victims. Given that this is the first research to 
explore the impact of social support on emotional violence, future research must continue to examine this relationship. Taken together, these findings support Richards and Branch's assertion that for female youth "friends, as opposed to parents, may function as 'guardians' over dating relationships" (p. 1555).

Consistent with prior research indicating that higher grades reduce the risk of both receiving abuse (Cleveland, Herrera, \& Stuewig, 2003; O'Keefe, 1998) and inflicting abuse for female youth (O'Keefe, 1998), female youth who reported higher average grades were less likely to engage in physical DV perpetration and experience physical DV victimization compared to those who reported lower average grades. Higher grades did not serve as a protective factor for emotional DV. In addition, concordant with previous research demonstrating that family violence is a strong predictor of later relationship violence (Gover, Kaukinen, \& Fox, 2008), female youth who experience more violence from parents are significantly more likely to perpetrate physical DV compared to female adolescents who experience less parental violence. Deviating from the physical DV perpetration model, female youth who experienced more family violence from parents were no more at risk for perpetrating emotional DV than female adolescents who experienced less parental violence. Also, non-White female youth are approximately twice as likely to perpetrate physical DV compared to White female youth and more than $35 \%$ more as likely to experience physical DV victimization compared to White female youth. This finding is in line with extant literature indicating that violent dating relationships are more prevalent among non-White female youth than White female youth (Connolly, Fiedlander, Pepler, Craig, \& Laporte, 2010; Foshee, Reyes, \& Ennett, 2010).

As in all research, this study is not without limitations. First, youth were not asked about their friends' attitudes toward or friends' involvement in DV. Previous research has indicated that teens that have friends who perpetrate DV are at an increased risk of also perpetrating such violence (Foshee et al., 2011). In addition, although this research encompassed both physical and emotional DV perpetration and victimization, an exploration of sexual aggression was beyond the scope of the current analyses. In addition, investigation of social support and the co-occurrence of perpetration and victimization were beyond the scope of the analysis. Future research should investigate how social support affects perpetration independent of experiences of victimization and vice versa. Specifically, are those who experience both perpetration and victimization different from those who experience only perpetration or only victimization? Finally, although this study used longitudinal data, only two time points were available for examination. Future research should attempt to further explore the impact of social support over the course of late childhood through adolescence to determine whether specific points in time are more responsive to social support from friends and/or parents.

\section{Implications and Future Research}

This research has demonstrated that increased social support from friends is related to a decrease in emotional DV victimization and physical and emotional DV perpetration among adolescent girls. This research has important implications for DV intervention and prevention programs. Recent Centers for Disease Control and Prevention (CDC; 2008) developed curricula for DV prevention asserts the importance of dyadic processes such as communication and emotional regulation, facets of socialization that are intrinsically female; however, such curricula fails to consider the potential impact of peer social support. This may represent a disconnection between what prevention/intervention experts think teens' need and what actually works for youth. 
One potential avenue for the integration of social support into DV programming is peer mentorship. Although they do not specifically focus on social support, Weisz and Black (2010) consider the potential merits of involving peer mentors/educators in DV intervention and prevention programs. Their findings highlight positive aspects of peer mentors such as their increased credibility among youth and the inherent access to nonviolent role models that peer education provides. In addition, peer educators allow youth to expand their circle of nonviolent friends who have knowledge concerning the signs of and resources for violence intervention. Importantly, Richards and Branch's (2012) prior study regarding the relationship between DV and social support highlights the importance of strengthening girls' peer networks especially girls who indicate low levels of social support from friends. Peer education provides an avenue for female youth, including adolescent victims and/or former perpetrators of DV, to empower themselves and expand their circle of friends in a prosocial way. In addition to peer education opportunities, Weisz and Black endorse alternative programs for youth engagement including the following: youth advisory boards, youth outreach programs, peer-counseling programs, and/or youth action programs. Such programs provide multiple ways in which girls can garner supportive peer relationships in an institutional context.

These findings also suggest that we need systematic research about why girls do not use their parents as social support providers. Many of the current DV prevention programs are focused on engaging educators and parents (Tharp, 2012); however, extant literature demonstrates that youth experiencing DV may not ask for help, but instead, parents may have to take the initiative and reach out to their teen. A recent study by Rothman, Miller, Terpeluk, Glauber, and Randel (2011) indicates that only about half (55\%) of parents talk to their teens about DV. Parents must be encouraged to breach the subject of DV, which for many parents is a difficult topic to talk about with their teen (Rothman et al., 2011). Similar to educational programming for youth, DV information must be widely disseminated to parents through the school system by including educational programming during parent nights and/or open houses. Exacerbating this issue, the presence of parental violence in the lives of girls and its impact on risk for DV victimization leaves many girls who are in most need of parental social support at a double disadvantage. DV curriculum must take parental violence into account and provide parents with information concerning their own/or their co-parent's interactions with their teen. In addition, community level campaigns against DV could include a parent component that not only provides parents with local resources for DV but for family violence as well.

As evidenced by the findings from this small but growing body of literature, the role that support from friends may play in reducing teen DV cannot be underscored. Therefore, it would appear wise to build on these promising findings by systematically expanding and integrating the concept of social support from friends into adolescent DV prevention and intervention programs.

\section{REFERENCES}

Abowitz, D., Knox, D., \& Zusman, M. (2010). Emotional abuse among undergraduates in romantic relationships. International Journal of Sociology of the Family, 36(2), 117-138.

Ackard, D., Neumark-Sztainer, D., \& Hannan, P. (2003). Dating violence among a nationally representative sample of adolescent girls and boys: Associations with behavioral and mental health. Journal of Gender-Specific Medicine, 6(3), 39-48.

Bennett, L., \& Fineran, S. (1998). Sexual and severe physical violence of high school students: Power beliefs, gender, and relationship. American Journal of Orthopsychiatry, 68, 645-652. 
Berenson, K., \& Anderson, S. (2006). Childhood physical and emotional abuse by a parent: Transference effects in adult interpersonal relations. Personality and Social Psychology Bulletin, 32(11), $1509-1522$.

Branch, K. (2008). The role of social support in the use of intimate partner violence: An exploration of the role of social support in heterosexual women's use of non-lethal intimate partner violence. Staarbrücken, Germany: VDM Verlag Dr. Muller e.K, Publishing.

Carlson, B. (1987). Dating violence: A research review and comparison with spouse abuse. Social Casework: The Journal of Contemporary Social Work, 16-23.

Carlson, B., McNutt, L., Choi, D., \& Rose, I. (2002). Intimate partner abuse and mental health: The role of social support and other protective factors. Violence Against Women, 8(6), 720-745.

Carver, K., Joyner, K., \& Udry, R. (2003). National estimates of adolescent romantic relationships. In P. Florsheim (Ed.), Adolescent romantic relationships and sexual behavior: Theory, research, and practical implications (pp. 23-56). Mahwah, NJ: LEA.

Centers for Disease Control and Prevention. (2008). Strategic direction for intimate partner violence prevention: Promoting respectful, nonviolent intimate partner relationships through individual, community, and societal change. Retrieved from www.cdc.gov/injury

Chen, Z., Williams, K., Fitness, J., \& Newton, N. (2008). When hurt will not heal: Exploring the capacity to relive social and physical pain. Psychological Science, 19(8), 789-795.

Clark, M., \& Ayers, M. (1993). Friendship expectations and friendship evaluations: Reciprocity and gender effects. Youth \& Society, 24, 299-313.

Cleveland, H., Herrera, V., \& Stuewig, J. (2003). Abusive males and abusive females in adolescent relationships: Risk factor similarity and dissimilarity and the role of relationship seriousness. Journal of Family Violence, 18(6), 325-339.

Colarossi, L., \& Eccles, J. (2003). Differential effects of support providers on adolescents' mental health. Social Work Research, 27, 19-30.

Connolly, J., Fiedlander, L., Pepler, D., Craig, W., \& Laporte, L. (2010). The ecology of adolescent dating aggression: Attitudes, relationships, media use, and socio-demographic risk factors. Journal of Aggression, Maltreatment, and Trauma, 19(5), 469-491.

Dobash, R., Dobash, R., Cavanagh, K., \& Lewis, R. (1998). Separate and intersecting realities: A comparison of men's and women's accounts of violence against women. Violence Against Women, 4, 382-414.

Eiser, J. R., Morgan, M., Gammage, P., \& Gray, E. (2011). Adolescent smoking: Attitudes, norms, and parental influence. British Journal of Social Psychology, 28(3), 193-202.

El-Bassel, N., Gilbert, L., Rajah, V., Foleno, A., \& Frye, V. (2001). Social support among women in methadone treatment who experience partner violence. Violence Against Women, 7(3), 246-274.

Foshee, V., Linder, G., Bauman, K., Langwick, S., Arriaga, X., Heath, J., . . Bangdiwala, S. (1996). The Safe Dates Project: Theoretical basis, evaluation, design, and selected baseline findings. American Journal of Preventive Medicine, 12(5), 39-47.

Foshee, V. A., Reyes, H. L. M., \& Ennett, S. T. (2010). Examination of sex and race differences in longitudinal predictors of adolescent dating violence perpetrations. Journal of Aggression, Maltreatment, and Trauma, 19(5), 492-516.

Foshee, V. A., Reyes, H. L. M., Ennett, S. T., Suchindran, C., Mathias, J., Karriker-Jaffe, K. J., . . . Benefield, T. S. (2011). Risk and protective factors distinguishing profiles of adolescent peer and dating violence perpetration. Journal of Adolescent Health, 48, 344-350.

Frey, C., \& Rothlisberger, C. (1996). Social support in healthy adolescents. Journal of Youth and Adolescence, 25, 17-31.

Gavin, H. (2011). Sticks and stones may break my bones: The effects of emotional abuse. Journal of Aggression, Maltreatment \& Trauma, 20(5), 503-529.

Giordano, P. C., Longmore, M. A., \& Manning, W. D. (2001). Toledo Adolescent Relationships Study (TARS): Wave 1, 2001 and Wave 2, 2002. ICPSR04679-v1. Ann Arbor, MI: Inter-university Consortium for Political and Social Research. http://dx.doi.org/10.3886/ICPSR04679.v1 
Giordano, P., Longmore, M., \& Manning, W. (2006). Gender and the meanings of adolescent romantic relationships: A focus on boys. American Sociological Review, 71(2), 260-287.

Gover, A. R., Kaukinen, C., \& Fox, K. A. (2008). The relationship between violence in the family of origin and dating violence among college students. Journal of Interpersonal Violence, 23(12), 1667-1693.

Haynie, D., Steffensmeier, D., \& Bell, K. (2007). Gender and serious violence: Untangling the role of friendship sex composition and peer violence. Youth Violence \& Juvenile Justice, 5(3), 235-253.

Howard, D., \& Wang, M. (2003). Risk profiles of adolescent girls who were victims of dating violence. Adolescence, 38, 1-14.

Krosnick, J. A., \& Judd, C. M. (1982). Transitions in social influence at adolescence: Who induces cigarette smoking? Developmental Psychology, 18, 359-368.

Kuttler, A., La Greca, A., \& Prinstein, M. (1999). Friendship qualities and social-emotional functioning of adolescents with close, cross-sex friendships. Journal of Research on Adolescence, 9, 339-366.

Larance, L., \& Porter, M. (2004). Observations from practice: Support group membership as a process of social capital formation among female survivors of domestic violence. Journal of Interpersonal Violence, 19(6), 676-690.

Lavoie, F., Robitaille, L., \& Hebert, M. (2000). Teen dating relationships and aggression: An exploratory study. Violence Against Women, 6(1), 6-36.

Lewis, S., \& Fremouw, W. (2001). Dating violence: A critical review of the literature. Clinical Psychology Review, 21(1), 105-127.

Linder, J., Crick, N., \& Collins, W. (2002). Relational aggression and victimization in young adults' romantic relationships: Associations with perceptions of parent, peer, and romantic relationships quality. Social Development, 11, 69-86.

Maccoby, E. (1998). The two sexes: Growing up apart, coming together. Cambridge, MA: Harvard University Press.

Makepeace, J. (1981). Courtship violence among college students. Family Relations, 30(1), 97-102.

Malecki, C., \& Demaray, M. (2003). What type of support do they need? Investigating student adjustment as related to emotional, informational, appraisal, and instrumental support. School Psychology Review, 18, 231-252.

Martino, S. C., Ellickson, P. L., \& McCaffrey, D. F. (2009). Multiple trajectories of peer and parental influence and their development of adolescent heavy drinking. Addictive Behaviors, 34(8), 693-700.

Muñoz-Rivas, M., Graña, J., O’Leary, K., \& González, M. (2007). Aggression in adolescent dating relationships: Prevalence, justification, and health consequences. Journal of Adolescent Health, 40, 298-304.

O'Keefe, M. (1997). Predictors of dating violence among high school students. Journal of Interpersonal Violence, 12, 546-568.

O'Keefe, M. (1998). Factors mediating the link between witnessing interparental violence and dating violence. Journal of Family Violence, 13(1), 39-57.

O'Leary, K. (1999). Developmental and affective issues in assessing and treating partner aggression. Clinical Psychology: Science and Practice, 6(4), 400-414.

O'Leary, K., \& Slep, A. (2012). Prevention of partner violence by focusing on behaviors of both young males and females. Prevention Science, 13, 329-339.

Raiford, J., Wingood, G., \& Diclemente, R. (2007). Prevalence, incidence, and predictors of dating violence: A study of African American female adolescents. Journal of Women's Health, 16(6), $822-832$.

Rees, C. (2010). Understanding emotional abuse. Archives of Disease in Childhood, 95(1), 59-67.

Richards, T. N., \& Branch, K. A. (2012). The relationship between social support adolescent dating violence: A comparison across genders. Journal of Interpersonal Violence, 27(8), 1540-1561.

Roberts, T., \& Klein, J. (2003). Intimate partner abuse and high-risk behavior in adolescents. Archives of Pediatrics \& Adolescent Medicine, 157(4), 375-380. 
Rothman, E. F., Miller, E., Terpluk, A., Glauber, A., \& Randal, J. (2011). The proportion of U.S. parents who talk with their adolescent children about dating violence. Journal of Adolescent Health, 49, 216-218.

Schonert-Reichl, K., \& Muller, J. (1996). Correlates of help-seeking in adolescence. Journal of Youth and Adolescence, 25(6), 705-731.

Sears, H. A., \& Byers, S. (2010). Adolescent girls' and boys' experiences of psychologically, physically, and sexually aggressive behaviors in their dating relationships: Co-occurrence and emotional reaction. Journal of Aggression, Maltreatment, \& Trauma, 19(5), 517-539.

Sears, H., Byers, E., Whelan, J., \& Saint-Pierre, M. (2006). "If it hurts you, then it is not a joke": Adolescents' ideas about girls' and boys' use and experience of abusive behavior in dating relationships. Journal of Interpersonal Violence, 21(9), 1191-1207.

Silverman, J. G., Raj, A., Mucci, L. A., \& Hathaway, J. E. (2001). Dating violence against adolescent girls and associated substance use, unhealthy weight control, sexual risk behaviour, pregnancy, and suicidality. The Journal of the American Medical Association, 286(5), 572-579.

Straus, M., \& Gelles, R. (1990). Physical violence in American families. New Brunswick, NJ: Transaction Publishers.

Swahn, M., Simon, T., Arias, I., \& Bossarte, R. (2008). Measuring sex differences in violence victimization and perpetration within date and same-sex peer relationships. Journal of Interpersonal Violence, 23(8), 1120-1138.

Tharp, A. (2012). Dating matters: The next generation of teen dating violence prevention. Preventive Science, 13, 398-401.

Thompson, M., Kaslow, N., Kingree, J., Rashid, A., Puett, R., Jacobs, D., \& Matthews, A. (2000). Partner violence, social support, and distress among inner-city African American women. American Journal of Community Psychology, 28(1), 127-143.

Underwood, M., \& Rosen, L. (2009). Gender, peer relations, and challenges for girlfriends and boyfriends coming together in adolescence. Psychology of Women Quarterly, 33, 16-20.

Vézina, J., \& Hébert, M. (2007). Risk factors for victimization in romantic relationships of young women: A review of empirical studies and implications for prevention. Trauma, Violence \& Abuse, 8, 33-66.

Warr, M. (2002). Companions in crime: The social aspects of criminal conduct. Cambridge, MA: Cambridge University Press.

Weisz, A. N., \& Black, B. M. (2010). Peer education and leadership in dating violence prevention: Strengths and challenges. Journal of Aggression, Maltreatment, and Trauma, 19(6), 641-660.

Zimmerman, B. (1995). Self-efficacy and educational development. In A. Bandura (Ed.), Selfefficacy in changing societies (pp. 202-231). New York, NY: Cambridge University Press.

Acknowledgments. This research uses data from TARS, whose PIs included Peggy C. Giordano, Monica A. Longmore, and Wendy D. Manning. The TARS is a longitudinal study and data collection is funded by multiple grants R01-HD036223-01 from the National Institute for Child Health and Human Development. Persons interested in obtaining data files from TARS should contact the Inter-Consortium for Social and Political Research, The University of Michigan, Institute for Social Research, P.O. Box 1248, Ann Arbor, MI 48106-1248, netmail@icpsr.umich.edu.

Correspondence regarding this article should be directed to Tara N. Richards, $\mathrm{PhD}$, School of Criminal Justice, University of Baltimore, 1420 North Charles Street, LAP 507, Baltimore, MD 21201. E-mail: trichards@ubalt.edu 